\title{
SYMMETRIC STABLE PROCESSES IN CONES
}

\author{
RODRIGO BAÑUELOS AND KRZYSZTOF BOGDAN
}

\begin{abstract}
We study exponents of integrability of the first exit time from generalized cones for conditioned rotation invariant stable Lévy processes. Along the way, we introduce the "spherical fractional Laplacian" and derive some of its spectral properties.
\end{abstract}

\section{INTRODUCTION}

For $x \in \mathbb{R}^{d} \backslash\{0\}$, we denote by $\theta(x)$ the angle between $x$ and the point $(0, \ldots, 0,1)$. The right circular cone of angle $\Theta \in(0, \pi)$ is the domain $\Gamma_{\Theta}=\left\{x \in \mathbb{R}^{d}: \theta(x)<\Theta\right\}$. The exact moments of integrability of the first exit time, $\tau_{\Gamma_{\ominus}}$, for both Brownian motion and conditioned Brownian motion from $\Gamma_{\Theta}$ have been extensively studied in the literature. These investigations began with the work of $\mathrm{D}$. L. Burkholder $[\mathrm{Bk}]$ who showed that there exists a constant $p(\Theta, d)$ such that for any $x \in \Gamma_{\Theta}, E_{x}\left(\tau_{\Gamma_{\Theta}}^{p}\right)<\infty$ if and only if $p<p(\Theta, d)$. This critical exponent, as it was shown by Burkholder, can be expressed in terms of zeros of confluent hypergeometric function. Extensions of the result were given by D. DeBlassie [De1], B. Davies and B. Zang [DZ], and R. Bañuelos and R. Smits [BS]. In particular, the results in [DZ] and [BS] provide the analogue of Burkholder's result for conditioned Brownian motion. In [De2], DeBlassie obtained a counterpart of Burkholder's result for symmetric stable processes in $\mathbb{R}^{2}$. DeBlassie's result was extended to all dimensions by P. Méndez-Hernández [M]. In [K3], T. Kulczycki gave results on the asymptotics of the critical exponent in right circular cones of decreasing aperture.

The purpose of the present paper is to obtain an analogue of the results in [DZ] and [BS] for conditioned symmetric stable processes in generalized cones and to extend the results in [De2] and [M] for the unconditioned processes to more general cones. Our method is motivated by, but different then, the method in [DZ]. The key step in our proof is to identify, for generalized

The first author was supported in part by NSF grant \# 9700585-DMS The second author was supported in part by KBN and by RTN contract HPRN-CT-200100273-HARP

2000 Mathematics Subject Classification: Primary 31B05, 60J45.

Key words and phrases: symmetric stable process, $\alpha$-harmonic function, cone, conditional process, exit time. 
cones, the Martin kernel with pole at infinity as a homogeneous function of degree $\beta \in[0, \alpha)$. Once this is done we use the scaling of the stable process and homogeneity of the kernel, together with tools related to the boundary Harnack principle of R. Song and J.-M. Wu [SW], to estimate the distribution of the exit time of the process from the cone. The exponent $\beta$ depends on the geometry of the cone and relates the considered problem to the asymptotics of harmonic functions at the vertex of the cone, see [B1] for boundary points of Lipschitz domains. We use $\beta$ to identify the critical moments of integrability of the exit time of the stable processes and the conditioned stable process from the cone as $\beta / \alpha$ and $(d-\alpha+2 \beta) / \alpha$, respectively, see Theorem 4.1 below. Our method should apply to other processes with scaling such as the censored processes studied in [BBC].

The paper is organized as follows. In $\S 2$ we recall the basic properties of the symmetric $\alpha$-stable processes and the definition of $\alpha$-harmonic functions. In $\S 3$ we recall the boundary Harnack principle of [SW] and state Theorem 3.2 and Theorem 3.3 which identify the Martin kernels at infinity and at 0 as homogeneous functions of degree $\beta$ and $\alpha-d-\beta$, respectively. We also give some explicit examples of cones where we identify these exponents in terms of the parameter $\alpha$. Theorems 3.2 and 3.3 are proved in $\S 6$. In $\S 4$ we obtain the above mentioned critical moments of integrability of the lifetime of the stable processes in the generalized cones. In $\S 5$ we study a "spherical fractional Laplacian", which is related in a natural way to the symmetric stable process. In the classical case of the Brownian motion, the spherical Laplacian plays a crucial role in understanding the moments of integrability of the corresponding process in cones. Indeed, the exponent of integration is obtained from the Dirichlet eigenvalues of the spherical Laplacian on the set of the sphere which generates the cones, see for example [BS]. While in the current case we were not able to obtain this precise relation to the exponent of integrability, nevertheless the spherical operator does provide some additional information. We also believe this operator may be useful in other settings and a more detailed study of its spectral properties will be of interest. In a sense, we give a polar coordinate decomposition of the fractional Laplacian, which as far as we know has not been given before.

\section{Preliminaries}

We begin by reviewing the notation used in this paper. By $|\cdot|$ we denote the Euclidean norm in $\mathbb{R}^{d}$. For $x \in \mathbb{R}^{d}, r>0$ and a set $A \subset \mathbb{R}^{d}$ we let $B(x, r)=$ $\left\{y \in \mathbb{R}^{d}:|x-y|<r\right\}$ and $\operatorname{dist}(A, x)=\inf \{|x-y|: y \in A\} . \bar{A}$ is the closure, and $A^{c}$ is the complement of $A$. We always assume Borel measurability of the considered sets and functions. The notation $c=c(\alpha, \beta, \ldots, \omega)$ means that $c$ is a constant depending only on $\alpha, \beta, \ldots, \omega$. Constants will always be 
(strictly) positive and finite. Throughout the paper we use the convention that $0 \cdot \infty=0$.

For the rest of the paper, unless stated otherwise, $\alpha$ is a number in $(0,2)$ and $d=1,2, \ldots$ By $\left(X_{t}, \mathbf{P}_{x}\right)$ we denote the standard $[\mathrm{BG}]$ rotation invariant ("symmetric") $\alpha$-stable, $\mathbb{R}^{d}$-valued Lévy process (that is, homogeneous, with independent increments), with index of stability $\alpha$ and characteristic function

$$
\mathbf{E}_{x} e^{i \xi\left(X_{t}-x\right)}=e^{-t|\xi|^{\alpha}}, \quad x \in \mathbb{R}^{d}, \quad \xi \in \mathbb{R}^{d}, \quad t \geq 0 .
$$

As usual, $\mathbf{E}_{x}$ denotes the expectation with respect to the distribution $\mathbf{P}_{x}$ of the process starting from $x \in \mathbb{R}^{d}$. The sample paths of $X_{t}$ are rightcontinuous with left limits almost surely. $\left(X_{t}, \mathbf{P}_{x}\right)$ is a Markov process with transition probabilities given by

$$
P_{t}(x, A)=\mathbf{P}_{x}\left(X_{t} \in A\right)=\int_{A} p(t ; x, y) d y
$$

and it is a strong Markov processes with respect to the standard filtration. For this and other basic properties, we refer the reader to [BG].

For an open set $U \subset \mathbb{R}^{d}$, we put $\tau_{U}=\inf \left\{t \geq 0 ; X_{t} \notin U\right\}$, the first exit time of $U$. Given $x \in \mathbb{R}^{d}$, the $\mathbf{P}_{x}$ distribution of $X_{\tau_{U}}$ is a subprobability measure on $U^{c}$ (probability measure if $U$ is bounded) called the $\alpha$-harmonic measure.

The scaling property of $X_{t}$ plays a key role in this paper. Namely, for $r>0$ we have that for every $x \in \mathbb{R}^{d}$ the $\mathbf{P}_{x}$ distribution of $X_{t}$ is the same as the $\mathbf{P}_{r x}$ distribution of $r^{-1} X_{r^{\alpha} t}$. In particular, the $\mathbf{P}_{x}$ distribution of $\tau_{U}$ is the same as the $\mathbf{P}_{r x}$ distribution of $r^{-\alpha} \tau_{r U}$. In short, $\tau_{r U}=r^{\alpha} \tau_{U}$ in distribution.

When $r>0,|x|<r$ and $B=B(0, r) \subset \mathbb{R}^{d}$, the corresponding $\alpha$-harmonic measure has the density function $P_{r}(x, \cdot)$ (the Poisson kernel) given by

$$
P_{r}(x, y)=C_{\alpha}^{d}\left[\frac{r^{2}-|x|^{2}}{|y|^{2}-r^{2}}\right]^{\alpha / 2}|y-x|^{-d} \quad \text { if } \quad|y|>r
$$

with $C_{\alpha}^{d}=\Gamma(d / 2) \pi^{-d / 2-1} \sin (\pi \alpha / 2)$, and 0 otherwise. The formula for the Poisson kernel for the exterior of the ball $\left\{y \in \mathbb{R}^{d}:|y-x|>r\right\}$ is similar. Namely, for $|x|>r$ we have

$$
\tilde{P}_{r}(x, y)=C_{\alpha}^{d}\left[\frac{|x|^{2}-r^{2}}{r^{2}-|y|^{2}}\right]^{\alpha / 2}|y-x|^{-d} \quad \text { if } \quad|y|<r
$$

and $\tilde{P}_{r}(x, y)=0$ if $|y| \geq r$. Both (1) and (2) can be found in [BGR].

Definition 2.1. We say that $f$ defined on $\mathbb{R}^{d}$ is $\alpha$-harmonic in an open set $D \subset \mathbb{R}^{d}$ if it satisfies the "mean value property"

$$
f(x)=\mathbf{E}_{x} f\left(X_{\tau_{U}}\right), \quad x \in U,
$$


for every bounded open set $U$ with closure contained in $D$. It is called regular $\alpha$-harmonic in $D$ if (3) holds for $U=D$.

In (3) we always assume that the expectation is absolutely convergent. If $D$ is unbounded then by the usual convention $\mathbf{E}_{x} u\left(X_{\tau_{D}}\right)=\mathbf{E}_{x}\left[u\left(X_{\tau_{D}}\right) ; \tau_{D}<\infty\right]$. By the strong Markov property a regular $\alpha$-harmonic function is $\alpha$-harmonic. The converse is not generally true as shown in [B2], [CS2]. An alternative description of $\alpha$-harmonic functions as those annihilating the fractional Laplacian

$$
\begin{aligned}
\Delta^{\alpha / 2} f(x) & =\mathcal{A}_{d, \alpha} \int_{\mathbb{R}^{d}} \frac{f(y)-f(x)-\nabla f(x) \cdot(y-x) \mathbf{1}_{\{|y-x|<1\}}}{|y-x|^{d+\alpha}} d y \\
& =\mathcal{A}_{d, \alpha} \lim _{\epsilon \rightarrow 0^{+}} \int_{B(x, \epsilon)^{c}} \frac{f(y)-f(x)}{|y-x|^{d+\alpha}} d y
\end{aligned}
$$

is given in [BB2]. Here and below, $\mathcal{A}_{d, \alpha}=\alpha 2^{\alpha-1} \pi^{-d / 2} \Gamma[(d+\alpha) / 2] / \Gamma(1-\alpha / 2)$ is an appropriate normalizing constant ([BG], $[\mathrm{L}])$. It follows from (1) and (3) that an $\alpha$-harmonic function $f$ in $D$ satisfies

$$
f(x)=\int_{|y-\theta|>r} P_{r}(x-\theta, y-\theta) f(y) d y, \quad x \in B(\theta, r),
$$

for every ball $B(\theta, r)$ of closure contained in $D$. In fact, the condition characterizes functions $\alpha$-harmonic in $D$. The integral in (4) is absolutely convergent and, by (1), $f$ is smooth in $D$ and

$$
\int_{\mathbb{R}^{d}} \frac{|f(y)|}{(1+|y|)^{d+\alpha}} d y<\infty .
$$

If, in addition, $f$ is nonnegative on $\mathbb{R}^{d}$ and nonzero in $D$, then it is positive in $D$, regardless of the connectedness of $D$. This is a consequence of Harnack inequality, see, e.g., [BB1].

\section{KeRnel FUnCtions}

The cones $\Gamma_{\Theta}$ described in the introduction are called right circular cones. By a generalized cone in $\mathbb{R}^{d}$ we shall mean in this paper an open set $\Gamma \subset \mathbb{R}^{d}$ with the property that if $x \in \Gamma$ and $r>0$ then $r x \in \Gamma$. If $0 \in \Gamma$ then $\Gamma=\mathbb{R}^{d}$. Otherwise, $\Gamma$ is characterized by its intersection with the unit sphere $\mathbb{S}^{d-1} \subset \mathbb{R}^{d}$. Namely, let $\Omega \neq \emptyset$ be a relatively open subset of $\mathbb{S}^{d-1}$. Without loosing generality in what follows, we assume that $\mathbf{1}=(0,0, \ldots, 0,1) \in \Omega$. The generalized cone spanned by $\Omega$ is then

$$
\Gamma=\Gamma^{\Omega}=\left\{x \in \mathbb{R}^{d}: x \neq 0 \text { and } x /|x| \in \Omega\right\} .
$$


Note that we do not impose any regularity properties on $\Omega$, in particular $\Gamma^{\Omega}$ may be disconnected.

For $n=0,1, \ldots$, we let $B_{n}=\left\{|x|<2^{n}\right\}$ and $\Gamma_{n}=\Gamma \cap B_{n}$.

The following result follows from [SW].

Lemma 3.1 (Boundary Harnack principle). There is a constant $C_{1}=$ $C_{1}(\Gamma, \alpha)$ such that for all functions $u, v \geq 0$ on $\mathbb{R}^{d}$ which vanish on $\Gamma^{c} \cap B_{1}$ and satisfy: $u(x)=v(x)$ for some $x \in \Gamma_{0}$,

$$
u(x)=\mathbf{E}_{x} u\left(X_{\tau_{\Gamma_{1}}}\right), \quad \text { for all } x \in \mathbb{R}^{d},
$$

and

$$
v(x)=\mathbf{E}_{x} v\left(X_{\tau_{\Gamma_{1}}}\right), \quad \text { for all } x \in \mathbb{R}^{d},
$$

we have

$$
C_{1}^{-1} v(x) \leq u(x) \leq C_{1} v(x), \quad x \in B_{0} .
$$

We note that by (6) and (7), $u$ and $v$ are regular $\alpha$-harmonic on $\Gamma_{1}$. By considering the regular $\alpha$-harmonic function $v(x)=\mathbf{P}_{x}\left[X_{\tau_{\Gamma_{1}}} \in B_{1}^{c}\right] \leq 1$ defined by the "boundary condition": $v=1$ on $B_{1}^{c}$ and $v=0$ on $B_{1} \cap \Gamma^{c}$, we see that (8) implies that every function $u$ satisfying the assumptions of Lemma 3.1 is bounded on $B_{0}$ and in fact,

$$
u(x) \leq C_{2} u(\mathbf{1}), \quad|x|<1,
$$

where $C_{2}=C_{1} / \mathbf{P}_{\mathbf{1}}\left[X_{\tau_{\Gamma_{1}}} \in B_{1}^{c}\right]<\infty$ depends only on $\alpha$ and $\Gamma$.

Recall that a function $h: \mathbb{R}^{d} \backslash\{0\} \rightarrow \mathbb{R}$ is homogeneous of degree $\beta$ if

$$
h(r x)=r^{\beta} h(x), \quad r>0, x \neq 0,
$$

or, equivalently, $h(x)=|x|^{\beta} h(x /|x|), x \neq 0$.

Theorem 3.2. There exists a unique nonnegative function $M$ on $\mathbb{R}^{d}$ such that $M(\mathbf{1})=1, M=0$ on $\Gamma^{c}$ and for every open bounded set $B \subset \Gamma$

$$
M(x)=\mathbf{E}_{x} M\left(X_{\tau_{B}}\right), \quad x \in \mathbb{R}^{d} .
$$

The function is locally bounded on $\mathbb{R}^{d}$ and homogeneous of degree $\beta=\beta(\Gamma, \alpha)$. That is,

$$
M(x)=|x|^{\beta} M(x /|x|), \quad x \neq 0 .
$$

Here, $\beta=0$ if $\Gamma^{c}$ is a polar set for $X_{t}$ and $0<\beta<\alpha$, otherwise.

The function $M$ will be called the Martin kernel with pole at infinity for $\Gamma$. The proof of Theorem 3.2 is given in $\S 6$ below. Note that if $\Gamma$ is a right circular cone then it is a Lipschitz domain. In this case, Theorem 3.2 follows from the uniqueness of the Martin representation of nonnegative $\alpha$-harmonic functions in Lipschitz domains [B2], [CS2], see also Example 4.1 in [BKN]. 
By (10) the function $M$ is regular $\alpha$-harmonic on every open bounded subset of $\Gamma$ and by Lemma 3.1 the decay rate of $M$, namely $|x|^{\beta}$ as $x \rightarrow 0$ along radii, is universal among all functions satisfying the assumptions of Lemma 3.1. By (9)

$$
M(x) \leq C_{2}|x|^{\beta}, \quad x \in \mathbb{R}^{d} .
$$

We shall now describe some examples of generalized cones where the exponent $\beta$ can be explicitly identified.

Example 3.1. Consider $\Gamma=\mathbb{R}^{d}$. The only nonnegative $\alpha$-harmonic functions on the whole of $\mathbb{R}^{d}$ are constants $[\mathrm{BKN}]$. Thus $M \equiv 1$ and $\beta=0$ for this cone.

Example 3.2. We put

$$
M(x)=\left\{\begin{array}{cll}
x_{d}^{\alpha / 2} & \text { if } & x_{d}>0 \\
0 & \text { if } & x_{d} \leq 0
\end{array}\right.
$$

where $x=\left(x_{1}, x_{2}, \ldots, x_{d}\right) \in \mathbb{R}^{d}$. Then $M$ is $\alpha$-harmonic on $\mathbb{R}^{d}+=\mathbb{R}^{d} \cap\left\{x_{d}>\right.$ $0\}$ by checking that $\Delta^{\alpha / 2} M(x)=0, x \in \mathbb{R}^{d}$ (we refer the reader to $[\mathrm{BBC}]$ for a related explicit calculation), see also [B2]. By Proposition 3.2, it is the Martin kernel at infinity for $\Gamma=\mathbb{R}^{d}$. In this case, $\beta=\alpha / 2$. (This rate of decay is characteristic for smooth domains [CS1], [K1].)

Example 3.3. Let $\alpha>1$ and consider the function $M(x)=\left|x_{d}\right|^{\alpha-1}, x \in \mathbb{R}^{d}$. Using the calculations in $[\mathrm{BBC}]$ one obtains $\Delta^{\alpha / 2} M(x)=0, x \in \mathbb{R}^{d}, x_{d} \neq 0$. Again by Proposition 3.2, $M$ is the Martin kernel at infinity for cone $\Gamma=$ $\mathbb{R}^{d} \backslash\left\{x_{d}=0\right\}$ and $\beta=\alpha-1$ in this case. For the same cone but for $\alpha \leq 1$, $M$ is the indicator function of $\mathbb{R} \backslash\left\{x_{d}=0\right\}$ since $\left\{x_{d}=0\right\}$ is a polar set if $\alpha \leq 1[\mathrm{~L}]$. This time we have $\beta=0$.

Let $\gamma, \Gamma$ be generalized cones in $\mathbb{R}^{d}$, and let $m, M$ be their respective Martin kernels with pole at infinity. If $\gamma \subset \Gamma$ then

$$
\mathbf{P}_{x}\left\{X_{\tau_{\gamma_{1}}} \in B(0,2)^{c}\right\} \leq \mathbf{P}_{x}\left\{X_{\tau_{\Gamma_{1}}} \in B(0,2)^{c}\right\}, \quad x \in \mathbb{R}^{d} .
$$

Here $\gamma_{1}=\gamma \cap B(0,2)$. By BHP there is a constant $c$ such that

$$
m(x) \leq c M(x), \quad x \in B(0,1) .
$$

We conclude that the respective homogeneity exponents satisfy $\beta(\gamma, \alpha) \geq$ $\beta(\Gamma, \alpha), 0<\alpha<2$. In fact, we have the following result.

Lemma 3.3. If $\gamma \subset \Gamma$ then $\beta(\gamma, \alpha) \geq \beta(\Gamma, \alpha)$. Furthermore, $\beta(\gamma, \alpha)>$ $\beta(\Gamma, \alpha)$ if and only if $\Gamma \backslash \gamma$ is a non-polar set.

Proof. We adopt the notation above. We only need to prove the second statement of the lemma. Let $\Gamma \backslash \gamma$ be nonpolar. We (falsely) assume that $\beta(\gamma)=\beta(\Gamma)$. There is a compact $K \subset \Gamma_{1} \backslash \gamma$ such that for some (hence every) 
starting point $x \in \Gamma_{1}$ we have with positive probability that $T_{K}<\tau_{\Gamma_{1}}$, hence $T_{K}=\tau_{\gamma_{1}}$. We clearly have

$$
M(x)>\mathbf{E}_{x}\left\{M\left(X_{\tau_{\gamma_{1}}}\right) \mathbf{1}_{\gamma}\left(X_{\tau_{\gamma_{1}}}\right)\right\}, \quad x \in \Gamma_{1} .
$$

Let

$$
a=\inf _{x \in \gamma} \frac{M(x)}{m(x)} .
$$

By (14) and our assumption $\beta(\gamma)=\beta(\Gamma)$ we have $a>0$. We define $H=$ $M \mathbf{1}_{\gamma}-a m$, and $h(x)=\mathbf{E}_{x} H\left(X_{\tau_{\gamma_{1}}}\right), x \in \mathbb{R}^{d}$. By (15) we have

$$
h(x)<M(x)-\operatorname{am}(x)=H(x), \quad x \in \gamma_{1} .
$$

We note that $H$ is homogeneous of degree $\beta(\gamma)=\beta(\Gamma)$ and nonnegative, so if $H(y)>0$ for some $y$ then $h(x)>0$ for all $x \in \gamma_{1}$. By BHP we have that $h \geq \varepsilon m$ on $B(0,1)$ with some $\varepsilon>0$, thus $H \geq \varepsilon m$ everywhere. In particular $M-a m \geq \varepsilon m$ or $a=\inf _{x \in \gamma} M(x) / m(x) \geq a+\varepsilon$, which is a contradiction. We conclude that $H \equiv 0$ or $M \mathbf{1}_{\gamma}=a m$. By the mean value property of $m$

$$
M(x)=\mathbf{E}_{x}\left\{M\left(X_{\tau_{\gamma_{1}}}\right) \mathbf{1}_{\gamma}\left(X_{\tau_{\gamma_{1}}}\right)\right\}, \quad x \in \gamma_{1} .
$$

This contradicts $(15)$ and thus $\beta(\gamma) \neq \beta(\Gamma)$.

On the other hand, if $\Gamma \backslash \gamma$ is a polar set then there is equality in (14) for $x \in \gamma$; in particular $\beta(\gamma)=\beta(\Gamma)$.

The above lemma gives a positive answer to the question of Ewa Damek whether the asymptotics of harmonic functions in "obtuse" cones is different than in the half-space, where $\beta=\alpha / 2$. On the other hand the proof of the lemma does not give quantitative information on $\beta(\gamma)-\beta(\Gamma)$ when $\Gamma \backslash \gamma$ is non-polar. We expect that spectral analysis of the spherical fractional Laplacian defined below may give such quantitative results.

By an application of Kelvin transform [B2] the function

$$
K(x)=|x|^{\alpha-d} M\left(x /|x|^{2}\right)=|x|^{\alpha-d-\beta} K(x /|x|), \quad x \neq 0,
$$

is $\alpha$-harmonic in $T \Gamma=\left\{x /|x|^{2} ; x \in \Gamma\right\}=\Gamma$, if $\Gamma \neq \mathbb{R}^{d}$. For completeness we put $K(0)=0$ so that $K=0$ on $\Gamma^{c}$. We call $K$ the Martin kernel at 0 for $\Gamma$, which is justified by the following theorem.

Theorem 3.4. $K$ given by (16) is the unique nonnegative function on $\mathbb{R}^{d}$ such that $K(\mathbf{1})=1, K=0$ on $\Gamma^{c}$ and for every open set $B \subset \Gamma$ such that $\operatorname{dist}(0, B)>0$,

$$
K(x)=\mathbf{E}_{x}\left\{K\left(X_{\tau_{B}}\right) ; \tau_{B}<\infty\right\}, \quad x \in \mathbb{R}^{d} .
$$

The proof of Theorem 3.4 is given in $\S 6$ below.

Example 3.4. In the context of Example 3.2 we obtain

$$
K(x)=\left\{\begin{array}{cl}
x_{d}^{\alpha / 2}|x|^{-d} & \text { if } \quad x_{d}>0 \\
0 & \text { if } \quad x_{d} \leq 0
\end{array}\right.
$$


On the other hand the Martin kernel at 0 for $\Gamma$ in Example 3.3 is $K(x)=$ $\left|x_{d}\right|^{\alpha-1}|x|^{2-\alpha-d}$ provided $\alpha>1$ (in particular $K(x)=1$ for $x \neq 0$, if $d=1$ ) and

$$
K(x)=\left\{\begin{array}{cc}
|x|^{\alpha-d}, & x_{d} \neq 0 \\
0, & x_{d}=0
\end{array}\right.
$$

provided $\alpha \leq 1$. (This is essentially M. Riesz kernel [L].)

\section{INTEGRABILITY OF EXIT TIMES}

We will write $\mathbf{P}_{x}^{0}$ and $\mathbf{E}_{x}^{0}$ for the probability and expectation associated with our stable process killed off the cone $\Gamma$ and conditioned by the Martin kernel $K$ of $\Gamma$ with the pole at 0 , as defined above. The process is a special case of the Doob h-process, in particular for any bounded or nonnegative function $f$ on $\Gamma$ we have

$$
\mathbf{E}_{x}^{0}\left\{f\left(X_{t}\right) ; \tau_{\Gamma}>t\right\}=\frac{1}{K(x)} \mathbf{E}_{x}\left\{K\left(X_{t}\right) f\left(X_{t}\right) ; \tau_{\Gamma}>t\right\} .
$$

Theorem 4.1. Let $\beta$ be the homogeneity degree of the Martin kernel $M$ of the cone $\Gamma$. For $p>0$ and $x \in \Gamma$ we have

$$
\mathbf{E}_{x} \tau_{\Gamma}^{p}<\infty \text { if and only if } p<\beta / \alpha,
$$

and

$$
\mathbf{E}_{x}^{0} \tau_{\Gamma}^{p}<\infty \quad \text { if and only if } \quad p<(d-\alpha+2 \beta) / \alpha .
$$

The proof of Theorem 4.1 follows immediately from the formula

$$
\mathbf{E} \tau^{p}=p \int_{0}^{\infty} t^{p-1} \mathbf{P}(\tau>t) d t, \quad p>0
$$

valid for any positive random variable $\tau$ on any probability space; and the following two lemmas.

Lemma 4.2. There is $C_{3}=C_{3}(\Gamma, \alpha)$ such that for all $t>0$ and $x \in \mathbb{R}^{d}$ satisfying $|x|<t^{1 / \alpha}$ we have

$$
C_{3}^{-1} M(x) t^{-\beta / \alpha} \leq \mathbf{P}_{x}\left\{\tau_{\Gamma}>t\right\} \leq C_{3} M(x) t^{-\beta / \alpha} .
$$

Proof. We first prove that there is $c_{1}=c_{1}(\Gamma, \alpha)$ such that

$$
c_{1}^{-1} M(x) \leq \mathbf{P}_{x}\left\{\tau_{\Gamma}>1\right\} \leq c_{1} M(x), \quad|x|<1 .
$$

This is a consequence of the boundary Harnack principle. Indeed, we let, as usual,

$$
\Gamma_{n}=\Gamma \cap B_{n}, \quad B_{n}=\left\{|x|<2^{n}\right\}, \quad n=0,1, \ldots,
$$

and we have

$$
\mathbf{P}_{x}\left\{\tau_{\Gamma}>1\right\} \leq \mathbf{P}_{x}\left\{\tau_{\Gamma_{1}}>1\right\}+\mathbf{P}_{x}\left\{\tau_{\Gamma 1}<\tau_{\Gamma}\right\}, \quad x \in \mathbb{R}^{d} .
$$


By the boundary Harnack principle

$$
\mathbf{P}_{x}\left\{\tau_{\Gamma 1}<\tau_{\Gamma}\right\} \leq C_{1} \mathbf{P}_{\mathbf{1}}\left\{\tau_{\Gamma 1}<\tau_{\Gamma}\right\} M(x), \quad|x|<1,
$$

where $C_{1}$ is the constant of Lemma 3.1. We let

$$
c_{2}=\inf _{v \in \Gamma_{1}} \int_{\Gamma \backslash \Gamma_{1}} \frac{\mathcal{A}_{d, \alpha}}{|y-v|^{d+\alpha}} d y .
$$

Clearly, $c_{2}>0$. We denote by $G$ the Green function of $\Gamma_{1}$ for our process $\left\{X_{t}\right\}$. By Ikeda-Watanabe formula ([IW]) and the boundary Harnack principle

$$
\begin{aligned}
\mathbf{P}_{x}\left\{\tau_{\Gamma_{1}}>1\right\} & \leq \mathbf{E}_{x} \tau_{\Gamma_{1}}=\int_{\Gamma_{1}} G_{\Gamma_{1}}(x, v) d v \\
& \leq c_{2}^{-1} \int_{\Gamma \backslash \Gamma_{1}} \int_{\Gamma_{1}} G_{\Gamma_{1}}(x, v) \frac{\mathcal{A}_{d, \alpha}}{|y-v|^{d+\alpha}} d v d y \\
& =c_{2}^{-1} P_{x}\left\{X_{\tau_{\Gamma_{1}}} \in \Gamma\right\} \leq c_{2}^{-1} C_{1} \mathbf{P}_{\mathbf{1}}\left\{X_{\tau_{\Gamma_{1}}} \in \Gamma\right\} M(x), \quad|x|<1 .
\end{aligned}
$$

This verifies the upper bound in (21). We then have

$$
\begin{aligned}
\mathbf{P}_{x}\left\{\tau_{\Gamma}>1\right\} & \geq \mathbf{P}_{x}\left\{\tau_{\Gamma_{3}}>1\right\} \\
& \geq \mathbf{E}_{x}\left[X_{\tau_{\Gamma_{1}}} \in B\left(4 \mathbf{1}, \operatorname{dist}\left(\mathbf{1}, \Gamma^{c}\right)\right) ; \mathbf{P}_{X_{\tau_{\Gamma_{1}}}}\left\{\tau_{\Gamma_{3}}>1\right\}\right] .
\end{aligned}
$$

Here, $B\left(4 \mathbf{1}, \operatorname{dist}\left(\mathbf{1}, \Gamma^{c}\right)\right)$ is the ball centered at $4 \mathbf{1}=(0,0, \ldots, 0,4) \in \Gamma$ and of radius $\operatorname{dist}\left(\mathbf{1}, \Gamma^{c}\right)$.

It is easy to verify that there is $c_{3}=c_{3}(\Gamma, \alpha)$ such that $P_{z}\left\{\tau_{\Gamma_{3}}>1\right\}>c_{3}$ for all $z \in B\left(4 \mathbf{1}, \operatorname{dist}\left(\mathbf{1}, \Gamma^{c}\right)\right)$. Thus, by the boundary Harnack principle, for $|x|<1$ we have

$$
\begin{aligned}
\mathbf{P}_{x}\left\{\tau_{\Gamma_{3}}>1\right\} & \geq c_{3} \mathbf{P}_{x}\left[X_{\tau_{\Gamma_{1}}} \in B\left(4 \mathbf{1}, \operatorname{dist}\left(\mathbf{1}, \Gamma^{c}\right)\right)\right] \\
& \geq c_{3} C_{1}^{-1} \mathbf{P}_{\mathbf{1}}\left[X_{\tau_{\Gamma_{1}}} \in B\left(4 \mathbf{1}, \operatorname{dist}\left(\mathbf{1}, \Gamma^{c}\right)\right)\right] M(x) .
\end{aligned}
$$

The proof of $(21)$ is complete.

To prove (20), we use the scaling of $\left\{X_{t}\right\},(21)$ and the homogeneity of $M$. For $t>|x|^{\alpha}$ the upper bound in (21) gives

$$
\begin{aligned}
\mathbf{P}_{x}\left\{\tau_{\Gamma}>t\right\} & =\mathbf{P}_{t^{-1 / \alpha} x}\left\{\tau_{\Gamma}>1\right\} \leq c_{1} M\left(t^{-1 / \alpha} x\right) \\
& =c_{1} M(x) t^{-\beta / \alpha} .
\end{aligned}
$$

Similarly, the lower bound in (21) gives $\mathbf{P}_{x}\left\{\tau_{\Gamma}>t\right\} \geq c_{1}^{-1} M(x) t^{-\beta / \alpha}$, completing the proof of Lemma 4.2.

Lemma 4.3. There is $C_{4}=C_{4}(\Gamma, \alpha)$ such that for all $t>0$ and $x \in \Gamma$ satisfying $|x|<t^{1 / \alpha}$ we have

$$
C_{4}^{-1} t^{(\alpha-d-2 \beta) / \alpha}|x|^{d-\alpha+2 \beta} \leq \mathbf{P}_{x}^{0}\left\{\tau_{\Gamma}>t\right\} \leq C_{4} t^{(\alpha-d-2 \beta) / \alpha}|x|^{d-\alpha+2 \beta} .
$$


Proof. For clarity we note that $M(x) / K(x)=|x|^{d-\alpha+2 \beta}, x \in \Gamma$, which is one factor in (22). As in the proof of Lemma 4.2, we first consider $t=1$ in (22). We have

$$
\mathbf{P}_{x}^{0}\left\{\tau_{\Gamma}>1\right\}=K(x)^{-1} \mathbf{E}_{x}\left\{K\left(X_{1}\right) ; \tau_{\Gamma}>1\right\} .
$$

To prove (22) for $t=1$ we only need to verify that there is $c_{1}=c_{1}(\Gamma, \alpha)$ such that

$$
c_{1}^{-1} M(x) \leq \mathbf{E}_{x}\left\{K\left(X_{1}\right) ; \tau_{\Gamma}>1\right\} \leq c_{1} M(x), \quad x \in \Gamma_{0} .
$$

We have

$$
\mathbf{E}_{x}\left\{K\left(X_{1}\right) ; \tau_{\Gamma}>1\right\}=\mathbf{E}_{x}\left\{K\left(X_{1}\right) ; \tau_{\Gamma_{3}} \leq 1, \tau_{\Gamma}>1\right\}+\mathbf{E}_{x}\left\{K\left(X_{1}\right) ; \tau_{\Gamma_{3}}>1\right\}
$$

By the $\alpha$-harmonicity of $K$ and Fatou's lemma

$$
K(x) \geq \mathbf{E}_{x} K\left(X_{\tau_{\Gamma_{3}}}\right), \quad x \in \mathbb{R}^{d} .
$$

Thus,

$$
\begin{aligned}
& \mathbf{E}_{x}\left\{K\left(X_{1}\right) ; \tau_{\Gamma_{3}}>1\right\} \\
\geq & \mathbf{E}_{x}\left\{\mathbf{E}_{X_{1}} K\left(X_{\tau_{\Gamma_{3}}}\right) ; \tau_{\Gamma_{3}}>1\right\}=\mathbf{E}_{x}\left\{K\left(X_{\tau_{\Gamma_{3}}}\right) ; \tau_{\Gamma_{3}}>1\right\} \\
\geq & \mathbf{E}_{x}\left\{\mathbf{E}_{X_{\tau_{\Gamma_{1}}}}\left\{K\left(X_{\tau_{\Gamma_{3}}}\right) ; \tau_{\Gamma_{3}}>1\right\} ; X_{\tau_{\Gamma_{1}}} \in B\left(4 \mathbf{1}, \operatorname{dist}\left(\mathbf{1}, \Gamma^{c}\right)\right)\right\} .
\end{aligned}
$$

There is $c_{2}=c_{2}(\Gamma, \alpha)$ such that $\mathbf{E}_{z}\left\{K\left(X_{\tau_{\Gamma_{3}}}\right) ; \tau_{\Gamma_{3}}>1\right\} \geq c_{2}$ for $z \in$ $B\left(4 \mathbf{1}\right.$, dist $\left.\left(\mathbf{1}, \Gamma^{c}\right)\right)$. By the boundary Harnack principle and the above

$$
\begin{aligned}
\mathbf{E}_{x}\left\{K\left(X_{1}\right) ; \tau_{\Gamma_{3}}>1\right\} & \geq c_{2} \mathbf{E}_{x}\left\{X_{\tau_{\Gamma_{1}}} \in B\left(4 \mathbf{1}, \operatorname{dist}\left(\mathbf{1}, \Gamma^{c}\right)\right)\right\} \\
& \geq c_{2} C_{1}^{-1} M(x) \mathbf{E}_{\mathbf{1}}\left\{X_{\tau_{\Gamma_{1}}} \in B\left(4 \mathbf{1}, \operatorname{dist}\left(\mathbf{1}, \Gamma^{c}\right)\right)\right\},
\end{aligned}
$$

which gives the lower bound in (23). To prove the upper bound we note that the transition density of the stable process killed off $\Gamma_{3}$ satisfies

$$
p_{1}^{\Gamma_{3}}(x, y) \leq c_{3} M(x) M(y), \quad x, y \in \mathbb{R}^{d},
$$

where $c_{3}=c_{3}(\Gamma, \alpha)$, as we shall see in Lemmas 5.2 and 5.3 below. It follows that

$$
\begin{aligned}
\mathbf{E}_{x}\left\{K\left(X_{1}\right) ; \tau_{\Gamma_{3}}>1\right\} & =\int_{\Gamma_{3}} p^{\Gamma_{3}}(1, x, y) K(y) d y \\
& \leq c_{3} \int_{\Gamma_{3}} M(x) M(y) K(y) d y \leq c_{4} M(x)
\end{aligned}
$$

with $c_{4}=c_{4}(\Gamma, \alpha)$. We also have

$$
\begin{aligned}
\mathbf{E}_{x}\left\{K\left(X_{1}\right) ; \tau_{\Gamma_{3}} \leq 1, \tau_{\Gamma}>1\right\} & =\mathbf{E}_{x}\left\{E\left\{K\left(X_{1}\right) \mid X_{\tau_{\Gamma_{3}}}\right\} ; \tau_{\Gamma_{3}} \leq 1, \tau_{\Gamma}>1\right\} \\
& \leq \mathbf{E}_{x}\left\{K\left(X_{\tau_{\Gamma_{3}}}\right) ; \tau_{\Gamma_{3}} \leq 1, \tau_{\Gamma}>1\right\} \\
& \leq \mathbf{E}_{x} K\left(X_{\tau_{\Gamma_{3}}}\right) \leq C_{1} \mathbf{E}_{1} K\left(X_{\tau_{\Gamma_{3}}}\right) M(x) \\
& \leq C_{1} K(\mathbf{1}) M(x)=C_{1} M(x) .
\end{aligned}
$$


The proof of (23) is complete.

To prove (22) we use (23), the scaling of $\left\{X_{t}\right\}$, and the homogeneity of $M$ and $K$. That is, for $t>0$ we have

$$
\begin{aligned}
\mathbf{P}_{x}^{0}\left\{\tau_{\Gamma}>t\right\} & =\frac{1}{K(x)} \mathbf{E}_{x}\left\{K\left(X_{t}\right) ; \tau_{\Gamma}>t\right\} \\
& =\frac{1}{K(x)} \mathbf{E}_{t^{-1 / \alpha} x}\left\{K\left(t^{1 / \alpha} X_{1}\right) ; \tau_{\Gamma}>1\right\} \\
& \leq c_{1}\left(t^{1 / \alpha}\right)^{\alpha-d-\beta} M\left(t^{-1 / \alpha} x\right) / K(x)=c_{1} t^{(\alpha-d-2 \beta) / \alpha}|x|^{d-\alpha+2 \beta} .
\end{aligned}
$$

The lower bound

$$
P_{x}^{0}\left\{\tau_{\Gamma}>t\right\} \geq c_{1}^{-1} t^{(\alpha-d-2 \beta) / \alpha}|x|^{d-\alpha+2 \beta}
$$

is proved similarly. This completes the proof of Lemma 3.3 under the assumption that (24) holds.

The estimate (24) is a direct consequence of the intrinsic ultracontractivity of the semigroup of the killed process $X_{t}$ which is valid for any bounded domain in $\mathbb{R}^{d}[\mathrm{~K} 2]$, see also [CS3]. This estimate, however, can be proved by other more elementary means, comp. $[\mathrm{R}]$. For a domain $D \subset \mathbb{R}^{d}$ let $s(x)=s_{D}(x)=\mathbf{E}_{x} \tau_{D}, x \in \mathbb{R}^{d}$. If $\sup \left\{s(x): x \in \mathbb{R}^{d}\right\}<\infty$ then $D$ is called Green bounded [BB2], [ChZ]. If the volume, $|D|$, of $D$ is finite and $D^{*}$ denotes the ball of same volume as $D$ centered at the origin, then (see, $[\mathrm{BLM}])$,

$$
\sup _{x \in \mathbb{R}^{d}} s_{D}(x) \leq s_{D^{*}}(0)=c_{d, \alpha}|D|^{\alpha / d}<\infty
$$

Thus domains of finite volume are Green bounded. It is also well known that there exist Green bounded domains of infinite volume.

Lemma 4.4. Suppose $D \subset \mathbb{R}^{d}$ is such that there exits a constant $C$ such that $s(x) \leq C_{0}$ for all $x \in \mathbb{R}^{d}$. Then for $n=1,2, \ldots$,

$$
\begin{gathered}
\mathbf{E}_{x} \tau_{D}^{n} \leq n ! C_{0}^{n-1} s(x), \quad x \in \mathbb{R}^{d}, \\
\mathbf{E}_{x} \exp \left(\varepsilon \tau_{D}\right)-1 \leq s(x) /\left(1-\varepsilon C_{0}\right), \quad x \in \mathbb{R}^{d}, \quad 0<\varepsilon<1 / C_{0},
\end{gathered}
$$

and

$$
\mathbf{P}_{x}\left\{\tau_{D}>t\right\} \leq \frac{1}{1-\varepsilon C_{0}} \frac{s(x)}{\exp (\varepsilon t)-1}, \quad x \in \mathbb{R}^{d}, t>0,0<\varepsilon<C_{0} .
$$

Proof. By the strong Markov property of $X_{t}$ we have for any $r>0$, 


$$
\begin{aligned}
\int_{r}^{\infty} \mathbf{P}_{x}\left\{\tau_{D}>t\right\} d t & =\int_{0}^{\infty} \mathbf{P}_{x}\left\{\tau_{D}>t+r\right\} \\
& =\int_{0}^{\infty} \mathbf{E}_{x}\left\{\mathbf{P}_{X_{r}}\left\{\tau_{D}>t\right\} ; \tau_{D}>r\right\} d t \\
& =\mathbf{E}_{x}\left\{\mathbf{1}_{\left\{\tau_{D}>r\right\}} \int_{0}^{\infty} \mathbf{P}_{X_{r}}\left\{\tau_{D}>t\right\} d t\right\} \\
& \leq C_{0} \mathbf{P}_{x}\left\{\tau_{D}>r\right\}
\end{aligned}
$$

For $n \geq 2$ we multiply both sides of the above inequality by $(n-1) r^{n-2}$ and integrate, applying Fubini's theorem on the left hand side, to obtain

$$
\mathbf{E}_{x} \tau_{D}^{n} \leq n C_{0} \mathbf{E}_{x} \tau_{D}^{n-1}
$$

The first asserted inequality follows by induction. The other two inequalities are even easier and are left to the reader.

Let $p_{t}^{D}(x, y)$ be the transition density of the process $X_{t}$ killed off $D$ :

$$
\mathbf{P}_{x}\left\{\tau_{D}>t, X_{t} \in A\right\}=\int_{A} p_{t}(x, y) d y .
$$

Clearly

$$
\begin{aligned}
p_{t}^{D}(x, y) & \leq p_{t}^{\mathbb{R}^{d}}(x, y)=t^{-d / \alpha} p_{1}^{\mathbb{R}^{d}}(x / t, y / t) \\
& \leq t^{-d / \alpha}(2 \pi)^{-d} \int_{\mathbb{R}^{d}} e^{-|\xi|^{\alpha}} d \xi=c t^{-d / \alpha}, \quad x, y \in D, t>0
\end{aligned}
$$

where $c=(2 \pi)^{-d} \omega_{d} \Gamma(d / \alpha) / \alpha$ and $\omega_{d}$ is the surface measure of the unit sphere in $\mathbb{R}^{d}$. Recall that

$$
\mathbf{P}_{x}\left\{\tau_{D}>t\right\}=\int_{D} p_{t}^{D}(x, y) d y .
$$

From these two observations and the semigroup property of $p_{t}^{D}(x, y)$ we have

$$
\begin{aligned}
p_{3 t}^{D}(x, y) & =\int_{D} p_{2 t}^{D}(x, z) p_{t}^{D}(z, y) d z \\
& =\int_{D}\left(\int_{D} p_{t}^{D}(x, w) p_{t}^{D}(w, z) d w\right) p_{t}^{D}(z, y) d z \\
& \leq \frac{c}{t^{d / \alpha}} \mathbf{P}_{x}\left\{\tau_{D}>t\right\} \mathbf{P}_{y}\left\{\tau_{D}>t\right\} .
\end{aligned}
$$

This together with Lemma 4.4 gives the following result.

Lemma 4.5. Under the assumptions of Lemma 4.4 there is $C=$ $C\left(\alpha, D, t_{0}, \varepsilon\right)$ such that

$$
p_{t}^{D}(x, y) \leq C e^{-2 \varepsilon t / 3} s(x) s(y), \quad x, y \in \mathbb{R}^{d}, \quad t>t_{0}, \quad 0<\varepsilon<C_{0} .
$$

Finally, the next lemma yields the estimate (24). 
Lemma 4.6. Let $s(x)=\mathbf{E}_{x} \tau_{\Gamma_{k}}, k=1,2, \ldots$ There is $c=c(\alpha, d, \Gamma, k)$ such that

$$
s(x) \leq c M(x), \quad x \in \mathbb{R}^{d} .
$$

Also,

$$
s(x) \geq c^{-1} M(x), x \in \Gamma_{k-1} .
$$

The lemma can be proved in a very similar way as Lemma 4.2 , by using the boundary Harnack principle and the Ikeda-Watanabe formula. We skip the details.

From (20) and (22) we immediately obtain the following result.

Corollary 4.7. There are constants $C_{3}$ and $C_{4}$ depending only on $\Gamma, \alpha$ and $d$, such that for all $x \in \Gamma$,

$$
\begin{aligned}
C_{3}^{-1}|x|^{\beta} M(x /|x|) & \leq \liminf _{t \rightarrow \infty} t^{\beta / \alpha} \mathbf{P}_{x}\left\{\tau_{\Gamma}>t\right\} \\
& \leq \limsup _{t \rightarrow \infty} t^{\beta / \alpha} \mathbf{P}_{x}\left\{\tau_{\Gamma}>t\right\} \\
& \leq C_{3}|x|^{\beta} M(x /|x|)
\end{aligned}
$$

and

$$
\begin{aligned}
C_{4}^{-1}|x|^{d-\alpha+2 \beta} & \leq \liminf _{t \rightarrow \infty} t^{(2 \beta+d-\alpha) / \alpha} \mathbf{P}_{x}^{0}\left\{\tau_{\Gamma}>t\right\} \\
& \leq \limsup _{t \rightarrow \infty} t^{(2 \beta+d-\alpha) / \alpha} \mathbf{P}_{x}^{0}\left\{\tau_{\Gamma}>t\right\} \\
& \leq C_{4}|x|^{d-\alpha+2 \beta}
\end{aligned}
$$

We also have the following "heat-kernel" version of this corollary. Recall that $p_{t}^{\Gamma}(x, y)$ are the transition densities (heat kernel) of $X_{t}$ killed off $\Gamma$.

Corollary 4.8. There is a constants $C_{5}$ depending only on $\Gamma, \alpha$ and $d$, such that for all $x, y \in \Gamma$,

$$
\begin{aligned}
C_{5}^{-1}|x|^{\beta}|y|^{\beta} M(x /|x|) M(y /|y|) & \leq \liminf _{t \rightarrow \infty} t^{(2 \beta+d) / \alpha} p_{t}^{\Gamma}(x, y) \\
& \leq \limsup _{t \rightarrow \infty} t^{(2 \beta+d) / \alpha} p_{t}^{\Gamma}(x, y) \\
& \leq C_{5}|x|^{\beta}|y|^{\beta} M(x /|x|) M(y /|y|)
\end{aligned}
$$

Proof. By (26) we have

$$
p_{3 t}^{\Gamma}(x, y) \leq \frac{c}{t^{d / \alpha}} \mathbf{P}_{x}\left\{\tau_{\Gamma}>t\right\} \mathbf{P}_{y}\left\{\tau_{\Gamma}>t\right\}
$$

with $c=(2 \pi)^{-d} \omega_{d} \Gamma(d / \alpha) / \alpha$. The upper bound follows from this and the previous result. 
For the lower bound, suppose first that $|x|,|y|<1$. By domain monotonicity, we have $p_{1}^{\Gamma}(x, y) \geq p_{1}^{\Gamma_{3}}(x, y)$. However, since $\Gamma_{3}$ is a bounded domain, it is intrinsically ultracontractive by [K2], [CS3]. Therefore

$$
p_{1}^{\Gamma_{3}}(x, y) \geq c_{1} \mathbf{P}_{x}\left\{\tau_{\Gamma_{3}}>1\right\} \mathbf{P}_{y}\left\{\tau_{\Gamma_{3}}>1\right\}
$$

From our proof of (21) it follows that for $|x|<1$ we have $\mathbf{P}_{x}\left\{\tau_{\Gamma_{3}}>1\right\} \geq$ $c_{2} M(x)$. From this we conclude that for $|x|,|y|<1$,

$$
p_{1}^{\Gamma}(x, y) \geq c_{3} M(x) M(y) .
$$

This inequality and scaling gives that for all $t>\max \left(|x|^{\alpha},|y|^{\alpha}\right)$,

$$
\begin{aligned}
p_{t}^{\Gamma}(x, y) & =t^{-d / \alpha} p_{1}^{\Gamma}\left(t^{-1 / \alpha} x, t^{-1 / \alpha} y\right) \\
& \geq c_{3} t^{-d / \alpha} M\left(t^{-1 / \alpha} x\right) M\left(t^{-1 / \alpha} y\right) \\
& =c_{3} t^{(-2 \beta-d) / \alpha} M(x) M(y) .
\end{aligned}
$$

The left hand side of (29) follows from this.

Corollaries 4.7 and 4.8 should be compared with the corresponding results for the Brownian motion in generalized cones ([BS] (1.5), (1.10) and (2.2)). However, the exact limits are computed in [BS]. It would be interesting to have the exact limits in the current setting as well. In addition to generalized cones, the above limits have been studied in [BDS], [Br], [Li], [LS] in the case of Brownian motion in parabolas and in other parabolic regions of the form $D=\left\{(x, y) \in \mathbb{R} \times \mathbb{R}^{d-1}: x>0,|y|<A x^{p}\right\}, 0<p<1$. The asymptotic behavior of the distribution of the exit time and of the heat kernel for such regions is shown to be subexponetial. It would be interesting to determine the behavior of the distribution of the exit time and the transition densities for stable processes in these regions. The scaling techniques we use here do not seem to apply.

\section{SPherical FRACTIONAL LAPlaCian}

In this section we study the action of $\Delta^{\alpha / 2}$ on homogeneous functions. We also introduce the corresponding spherical operator and give some of its properties which may be of importance in studying the exponent $\beta(\Gamma, \alpha)$.

We first consider an arbitrary function $\phi$ on $\mathbb{R}^{d}$ such that $\Delta^{\alpha / 2} \phi(\mathbf{1})$ is well defined. This is satisfied if

$$
\int_{\mathbb{R}^{d}} \frac{|\phi(y)|}{(1+|y|)^{d+\alpha}} d y<\infty
$$

and, say, $|\phi(\mathbf{1}+x)-\phi(\mathbf{1})-\nabla \phi(\mathbf{1}) \cdot x| \leq c|x|^{2}$ for $|x|<1$, e.g. $\phi$ is $C^{2}$ at $\mathbf{1}$.

Lemma 5.1. We have

$$
\lim _{\Theta \rightarrow 0^{+}} \int_{\mathbb{R}^{d} \backslash \Gamma_{\Theta}} \frac{(y-\mathbf{1}) \mathbf{1}_{\{|y-\mathbf{1}|<1\}}}{|y-\mathbf{1}|^{d+\alpha}} d y=0
$$


and

$$
\Delta^{\alpha / 2} \phi(\mathbf{1})=\mathcal{A}_{d, \alpha} \lim _{\Theta \rightarrow 0^{+}} \int_{\mathbb{R}^{d} \backslash \Gamma_{\Theta}} \frac{\phi(y)-\phi(\mathbf{1})}{|y-\mathbf{1}|^{d+\alpha}} d y .
$$

The proof of (31) is somewhat tedious (because $\Gamma_{\Theta}$ is not symmetric about 1) but elementary and will be omitted. The formula (32) follows from (31) immediately.

Let $\phi$ be also homogeneous of degree $\gamma ; \phi(x)=|x|^{\gamma} \phi(x /|x|), x \neq 0$. In view of (30) we will only consider $-d<\gamma<\alpha$.

By (32) and polar coordinates

$$
\begin{aligned}
\Delta^{\alpha / 2} \phi(\mathbf{1}) & =\mathcal{A}_{d, \alpha} \lim _{\Theta \rightarrow 0^{+}} \int_{\mathbb{R}^{d} \backslash \Gamma_{\Theta}} \frac{\phi(y)-\phi(\mathbf{1})}{|y-\mathbf{1}|^{d+\alpha}} d y \\
& =\mathcal{A}_{d, \alpha} \lim _{\Theta \rightarrow 0^{+}} \int_{\mathbb{S}^{d-1} \backslash \Gamma_{\Theta}} \sigma(d \theta) \int_{0}^{\infty} r^{d-1} \frac{\phi(\theta) r^{\gamma}-\phi(\mathbf{1})}{|r \theta-\mathbf{1}|^{d+\alpha}} d y \\
& =\mathcal{A}_{d, \alpha} \lim _{\Theta \rightarrow 0^{+}} \int_{\mathbb{S}^{d-1} \backslash \Gamma_{\Theta}}\left[\phi(\theta) u_{\gamma}\left(\theta_{d}\right)-\phi(\mathbf{1}) u_{0}\left(\theta_{d}\right)\right] \sigma(d \theta) .
\end{aligned}
$$

Here

$$
u_{\gamma}(t)=\int_{0}^{\infty} r^{d+\gamma-1}\left(r^{2}-2 r t+1\right)^{-(d+\alpha) / 2} d r, \quad-1 \leq t \leq 1
$$

(we drop $d$ and $\alpha$ from the notation); and (33) holds because

$$
\int_{0}^{\infty} r^{d+\gamma-1}|r \theta-\mathbf{1}|^{-d-\alpha} d r=\int_{0}^{\infty} r^{d+\gamma-1}\left(r^{2}-2 r \theta_{d}+1\right)^{-(d+\alpha) / 2} d r .
$$

Lemma 5.2. For every $-1 \leq t \leq 1$

$$
u_{\gamma}(t)=u_{\alpha-d-\gamma}(t), \quad-d<\gamma<\alpha
$$

and the function $\gamma \mapsto u_{\gamma}(t)$ is increasing on $[(\alpha-d) / 2, \alpha)$ with $u_{\alpha-}(t)=-\infty$.

Proof. Let $-1 \leq t \leq 1$. By a change of variable

$$
\begin{aligned}
u_{\gamma}(t) & =\int_{0}^{1} r^{d+\gamma-1}\left(r^{2}-2 r t+1\right)^{-(d+\alpha) / 2} d r \\
& +\int_{0}^{\infty} s^{-d-\gamma-1}\left(s^{-2}-2 s^{-1} t+1\right)^{-(d+\alpha) / 2} d s \\
& =\int_{0}^{1} \frac{1}{r}\left(r^{d+\gamma}+r^{\alpha-\gamma}\right)\left(r^{2}-2 r t+1\right)^{-(d+\alpha) / 2} d r
\end{aligned}
$$


which proves (35). Let $(\alpha-d) / 2 \leq \gamma_{1}<\gamma_{2}<\alpha$. We have

$$
\begin{aligned}
u_{\gamma_{2}}(t)-u_{\gamma_{1}}(t) & =\int_{0}^{1} \frac{1}{r}\left(r^{d+\gamma_{2}}+r^{\alpha-\gamma_{2}}-r^{d+\gamma_{1}}-r^{\alpha-\gamma_{1}}\right)\left(r^{2}-2 r t+1\right)^{-(d+\alpha) / 2} d r \\
(36) & =\int_{0}^{1} \frac{1}{r}\left(r^{\gamma_{1}}-r^{\gamma_{2}}\right)\left(r^{\alpha-\gamma_{2}-\gamma_{1}}-r^{d}\right)\left(r^{2}-2 r t+1\right)^{-(d+\alpha) / 2} d r>0,
\end{aligned}
$$

because $\alpha-\gamma_{2}-\gamma_{1}<\alpha-2 \gamma_{1} \leq \alpha-(\alpha-d)=d$. To verify that $u_{\alpha-}(t)=-\infty$ we note that for $\gamma \rightarrow \alpha$

$$
\begin{aligned}
u_{\gamma}(t) & \geq \int_{1}^{\infty} r^{d+\gamma-1}\left(r^{2}-2 r t+1\right)^{-(d+\alpha) / 2} d r \\
& \uparrow \int_{1}^{\infty} r^{d+\alpha-1}\left(r^{2}-2 r t+1\right)^{-(d+\alpha) / 2} d r \\
& \geq \int_{1}^{\infty} r^{d+\alpha-1}(r+1)^{-d-\alpha} d r=\infty .
\end{aligned}
$$

The proof is complete.

By (33) for $\phi$ homogeneous of degree $\gamma$ we have

$$
\begin{aligned}
\Delta^{\alpha / 2} \phi(\mathbf{1}) & =\mathcal{A}_{d, \alpha} P . V . \int_{\mathbb{S}^{d-1}}[\phi(\theta)-\phi(\mathbf{1})] u_{0}\left(\theta_{d}\right) \sigma(d \theta) \\
& +\mathcal{A}_{d, \alpha} \int_{\mathbb{S}^{d-1}} \phi(\theta)\left[u_{\gamma}\left(\theta_{d}\right)-u_{0}\left(\theta_{d}\right)\right] \sigma(d \theta)
\end{aligned}
$$

The principal value integral (P.V.) above is understood as in (33). We note that the second integral in (37) vanishes if $\phi$ is homogeneous of degree 0 and the first one vanishes whenever $\phi$ is constant on the unit sphere. The observation can be used to show that the integrals converge and the second one converges absolutely (conf. Lemma 5.2), a fact that can be also verified by a detailed inspection of $u_{\gamma}$ at $t=1$. A formula similar to (37) clearly holds for every vector $\eta \in \mathbb{S}^{d-1}$ :

$$
\begin{aligned}
\Delta^{\alpha / 2} \phi(\eta) & =\mathcal{A}_{d, \alpha} P . V \cdot \int_{\mathbb{S}^{d-1}}[\phi(\theta)-\phi(\eta)] u_{0}(\theta \cdot \eta) \sigma(d \theta) \\
& +\mathcal{A}_{d, \alpha} \int_{\mathbb{S}^{d-1}} \phi(\theta)\left[u_{\gamma}(\theta \cdot \eta)-u_{0}(\theta \cdot \eta)\right] \sigma(d \theta)
\end{aligned}
$$

where $\theta \cdot \eta$ denotes the usual scalar product of $\theta$ and $\eta$. The operator

$$
\begin{aligned}
\Delta_{\mathbb{S}^{d-1}}^{\alpha / 2} \phi(\eta) & =\mathcal{A}_{d, \alpha} P . V \cdot \int_{\mathbb{S}^{d-1}}[\phi(\theta)-\phi(\eta)] u_{0}(\theta \cdot \eta) \sigma(d \theta) \\
& =\mathcal{A}_{d, \alpha} \lim _{\varepsilon \rightarrow 0^{+}} \int_{\mathbb{S}^{d-1} \cap\{1-\theta \cdot \eta>\varepsilon\}}[\phi(\theta)-\phi(\eta)] u_{0}(\theta \cdot \eta) \sigma(d \theta) .
\end{aligned}
$$

will be called the spherical fractional Laplacian. The second integral in (38) will be called the "radial" part and denoted $R_{\gamma} \phi$ below. 
Remark 1. By Lemma 5.2 and (36) with $\gamma_{2}=\alpha-d$ and $\gamma_{1}=0$ we see that $\phi(x)=|x|^{\gamma}$ is $\alpha$-harmonic on $\mathbb{R}^{d} \backslash\{0\}$ if and only if $\gamma=\alpha-d$.

We will be concerned with nonnegative definiteness of the kernel $u_{\gamma}(\theta \cdot \eta)$. We first consider dimension $d=2$ and we will identify $\mathbb{R}^{2}$ with the complex plane $\mathbb{C}$. By a rotation, $O x_{2}$ axis in $\mathbb{R}^{2}$ will be identified with $\Re z$ axis in $\mathbb{C}$ so that $\mathbf{1} \in \mathbb{R}^{2}$ is now represented by $1 \in \mathbb{C}$. $\theta \in \mathbb{S}_{1}$ will be replaced by $e^{i \theta}$, $\theta \in \mathbb{T} \sim[0,2 \pi)$ in formulas. With this identification in mind we define

$$
u_{\gamma}^{(2)}(\theta)=\int_{0}^{\infty} r^{2+\gamma-1}\left|r e^{i \theta}-1\right|^{-2-\alpha} d r .
$$

For $r \in[0,1)$ and $\eta>0$ let $f(\theta)=\left|r e^{i \theta}-1\right|^{-\eta}=\sum_{-\infty}^{\infty} c_{n} e^{i n \theta}, \theta \in[0,2 \pi)$.

Lemma 5.3. For every $\eta>0$ and $r \in(0,1)$ we have $c_{n}>0, n \in \mathbb{Z}$.

Proof. Let $z=r e^{i \theta}$. We have $|z|<1$ and $\log (1-z)=-\sum_{n=1}^{\infty} x^{n} / n$, thus

$$
\log |1-z|=-\Re \sum_{n=1}^{\infty} r^{n} e^{i n \theta} / n=-\sum_{n=-\infty, n \neq 0}^{\infty} r^{|n|} e^{i n \theta} / n .
$$

Since $|1-z|^{-\eta}=\exp (-\eta \log |1-z|)$, the sequence $\left\{c_{n}=c_{n}(r)\right\}$ is the convolution exponent of the sequence $\left\{\mathbf{1}_{\{n \neq 0\}} \eta r^{|n|} /(2 n)\right\}_{-\infty}^{\infty}$. The latter sequence is nonnegative and so $\left\{c_{n}\right\}$ is positive $\left(c_{0} \geq 1\right)$.

Let $\phi$ be a test function and let $\eta, \theta \in[0,2 \pi)$. We consider the operator

$$
R_{\gamma}^{(2)} \phi(\eta)=\int_{0}^{2 \pi} \phi(\omega)\left[u_{\gamma}^{(2)}(\theta-\eta)-u_{0}^{(2)}(\theta-\eta)\right] d \theta
$$

For $\gamma \geq 0$ we have that $R_{\gamma}^{(2)} \geq 0$ in the sense that

$$
\int_{0}^{2 \pi} R_{\gamma}^{(2)} \phi(\theta) \overline{\phi(\theta)} d \theta \geq 0
$$

for all such $\phi$. In fact we have the following result.

Lemma 5.4. If $(\alpha-d) / 2 \leq \gamma_{1} \leq \gamma_{2}<\alpha$ then $R_{\gamma_{2}}^{(2)}-R_{\gamma_{1}}^{(2)} \geq 0$.

Proof. Let $\phi(\theta)=\sum_{n=-\infty}^{\infty} a_{n} e^{i n \theta} \in L^{2}(0,2 \pi)$. By (36) and Lemma 5.3 with $\eta=d+\alpha$ we have

$$
\begin{aligned}
& \int_{0}^{2 \pi}\left[R_{\gamma_{2}}^{(2)}-R_{\gamma_{1}}^{(2)}\right] \phi(\theta) \overline{\phi(\theta)} d \theta \\
= & \int_{0}^{1} \frac{1}{r}\left(r^{\gamma_{1}}-r^{\gamma_{2}}\right)\left(r^{\alpha-\gamma_{2}-\gamma_{1}}-r^{d}\right) \int_{0}^{2 \pi} \int_{0}^{2 \pi} \sum_{-\infty}^{\infty} c_{n} e^{i n(\theta-\omega)} \phi(\omega) \overline{\phi(\theta)} d \omega d \theta d r \\
= & 4 \pi^{2} \sum_{-\infty}^{\infty}\left|a_{n}\right|^{2} \int_{0}^{1} \frac{1}{r}\left(r^{\gamma_{1}}-r^{\gamma_{2}}\right)\left(r^{\alpha-\gamma_{2}-\gamma_{1}}-r^{d}\right) c_{n}(r) d r>0,
\end{aligned}
$$

unless $\phi=0$ a.e. (comp. the proof of Lemma 5.2). 
Theorem 5.5. $\left\{R_{\gamma}, \gamma \in[0, \alpha)\right\}$ is an increasing family of nonnegative definite operators on $L^{2}\left(\mathbb{S}^{d-1}, \sigma\right), d \geq 2$.

Proof. Let $(\alpha-d) / 2 \leq \gamma_{1} \leq \gamma_{2}<\alpha$. We only need to prove that $K=$ $R_{\gamma_{2}}-R_{\gamma_{2}}$ is nonnegative definite. For dimension $d=2$ this is proved above under a slightly different notation. We now let $d \geq 3$. For a test function $\phi$ on $\mathbb{S}^{d-1}$

$$
K \phi(\eta)=\mathcal{A}_{d, \alpha} \int_{\mathbb{S}^{d-1}} \phi(\theta)\left[u_{\gamma_{2}}(\theta \cdot \eta)-u_{\gamma_{1}}(\theta \cdot \eta)\right] \sigma(d \theta),
$$

compare (38). $K$ has the form of spherical convolution hence it is diagonalized by spherical harmonics;

$$
K Y_{m}=K_{m} Y_{m}, \quad m=0,1, \ldots,
$$

where $Y_{m}$ is any spherical harmonics of degree $m$, and, by Funk-Hecke formula ([E], (11.4.24), page 248 or [Ru], (2.15)-(2.19), page 10),

$$
K_{m}=c_{m} \int_{-1}^{1}\left[u_{\gamma_{2}}(t)-u_{\gamma_{1}}(t)\right] C_{m}^{d / 2-1}(t)\left[1-t^{2}\right]^{(d-3) / 2} d t
$$

where

$$
c_{m}=\frac{2 \pi^{(d-1) / 2}}{\Gamma((d-1) / 2)) C_{m}^{d / 2-1}(1)}
$$

and $C_{m}^{d / 2-1}$ are the Gegenbauer (ultraspherical) polynomials ([E], (10.9), page 174).

We only need to verify that $K_{m} \geq 0, m=0,1, \ldots$ By (36) it is enough to prove that for every $r \in[0,1)$ the spherical convolution

$$
k \phi(\eta)=\int_{\mathbb{S}^{d-1}} \phi(\theta)\left[r^{2}-2 r \theta \cdot \eta+1\right]^{-(d+\alpha) / 2} \sigma(d \theta)
$$

has nonnegative eigenvalues

$$
k_{m}=c_{m} \int_{-1}^{1}\left[r^{2}-2 r t+1\right]^{-(d+\alpha) / 2} C_{m}^{d / 2-1}(t)\left[1-t^{2}\right]^{(d-3) / 2} d t .
$$

By Rodrigues' formula ([E], (10.9.11), page 175),

$$
C_{m}^{d / 2-1}(t)=(-1)^{m} d_{m}\left(1-t^{2}\right)^{(3-d) / 2} \frac{d^{m}}{d t^{m}}\left[\left(1-t^{2}\right)^{m+(d-1) / 2}\right]
$$

where

$$
d_{m}=\frac{(d-2)_{m}}{2^{m} m !(d / 2-1 / 2)_{m}}
$$


and we used the notation $(\lambda)_{k}=\lambda(\lambda+1) \ldots(\lambda+k-1)$. Integrating by parts we obtain

$$
\begin{aligned}
k_{m} & =(-1)^{m} c_{m} d_{m} \int_{-1}^{1}\left[r^{2}-2 r t+1\right]^{-(d+\alpha) / 2} \frac{d^{m}}{d t^{m}}\left[\left(1-t^{2}\right)^{m+(d-1) / 2}\right] d t \\
& =c_{m} d_{m} \int_{-1}^{1} \frac{d^{m}}{d t^{m}}\left[r^{2}-2 r t+1\right]^{-(d+\alpha) / 2}\left(1-t^{2}\right)^{m+(d-1) / 2} d t
\end{aligned}
$$

By induction we easily check that

$$
\frac{d^{m}}{d t^{m}}\left[r^{2}-2 r t+1\right]^{-(d+\alpha) / 2}=2^{m}\left(\frac{d+\alpha}{2}\right)_{m} r^{m}\left[r^{2}-2 r t+1\right]^{-(d+\alpha) / 2-m} .
$$

Thus

$k_{m}=2^{m}\left(\frac{d+\alpha}{2}\right)_{m} c_{m} d_{m} r^{m} \int_{-1}^{1}\left[r^{2}-2 r t+1\right]^{-(d+\alpha) / 2-m}\left(1-t^{2}\right)^{m+(d-1) / 2} d t>0$.

The proof is complete.

For clarity we note that the operator $\Delta_{\mathbb{S}^{d-1}}^{\alpha / 2}$ is negative semi-definite on $L^{2}\left(\mathbb{S}^{d-1}, \sigma\right)$. Namely, for test functions $\phi$ we have

$$
\int_{\mathbb{S}^{d-1}} \Delta_{\mathbb{S}^{d-1}}^{\alpha / 2} \phi(\eta) \overline{\phi(\eta)} \sigma(d \eta)=-\frac{1}{2} \mathcal{A}_{d, \alpha} \int_{\mathbb{S}^{d-1} \times \mathbb{S}^{d-1}}[\phi(\theta)-\phi(\eta)]^{2} u_{0}(\theta \cdot \eta) \sigma(d \theta) \sigma(d \eta),
$$

which is negative unless $\phi$ is constant on $\mathbb{S}^{d-1}$. The proof of the above equality using the approximation (39) is standard and will be omitted.

Example 5.1. Let $d=2$ and $\alpha=1$. The kernel of $\Delta_{\mathbb{S}^{d-1}}^{\alpha / 2}$ is

$$
u_{0}(t)=\int_{0}^{1}(r+1)\left(r^{2}-2 r t+1\right)^{-3 / 2} d r, \quad|t| \leq 1,
$$

see the proof of Lemma 5.2. We use Euler change of variable: $\sqrt{r^{2}-2 r t+1}=$ $x-r$, to get

$$
u_{0}(t)=2 \int_{1}^{1+\sqrt{2(1-t)}} \frac{x^{2}+2 x-2 t-1}{\left(x^{2}-2 t x+1\right)^{2}} d x .
$$

The primitive function of the last integrand is $-(x+1) /\left(x^{2}-2 t x+1\right)$, which yields

$$
u_{0}(t)=\frac{1}{1-t}
$$

Thus, for a test function $\phi$ on $\mathbb{S}^{1}$ we have

$$
\Delta_{\mathbb{S}^{1}}^{1 / 2} \phi(\eta)=\frac{1}{2 \pi} P . V \cdot \int_{\mathbb{S}^{1}} \frac{\phi(\theta)-\phi(\eta)}{1-\theta \cdot \eta} \sigma(d \theta) .
$$

In particular, if $\phi_{m}(\theta)=\left(\theta_{1}+i \theta_{2}\right)^{m}, \theta=\left(\theta_{1}, \theta_{2}\right), n=0, \pm 1, \pm 2, \ldots$ (exponential basis on the torus), then, after a calculation, we obtain $\Delta_{\mathbb{S}^{1}}^{1 / 2} \phi_{m}=$ $-|m| \phi_{m}$. Similarly, trigonometric functions diagonalize $\Delta_{\mathbb{S}^{1}}^{\alpha / 2}$. 
We conclude this section with a variant of Funk-Hecke formula for $\Delta_{\mathbb{S} d-1}^{\alpha / 2}$ when $d \geq 3$. For every spherical harmonics $Y_{m}$ of order $m=0,1, \ldots$ we have

$$
\Delta_{\mathbb{S}^{d-1}}^{\alpha / 2} Y_{m}=\lambda_{m} Y_{m}
$$

where

$$
\lambda_{m}=c_{m} \int_{-1}^{1}\left[C_{m}^{d / 2-1}(t)-C_{m}^{d / 2-1}(1)\right] u_{0}(t)\left[1-t^{2}\right]^{(d-3) / 2} d t,
$$

and $c_{n}$ is given in (43). We recall a formula for the Gegenbauer polynomials ([E], (10.9.18), page 175)

$$
C_{m}^{\kappa}(\cos \theta)=\sum_{n=0}^{m} \frac{(\kappa)_{n}(\kappa)_{m-n}}{n !(m-n) !} \cos (m-2 n) \theta .
$$

$\lambda_{0}=0$ because $C_{0}^{d / 2-1} \equiv 0$. For $m>0$ we note that $C_{m}^{d / 2-1}$ attains its maximum at $t=1$, and so $\lambda_{m}<0$. The proof of (45) follows from the usual Funk-Hecke formula via the approximation (39).

A further study of spectral properties of $\Delta_{\mathbb{S} d-1}^{\alpha / 2}$ in relation to the increasing operator family $\left\{R_{\gamma}, 0 \leq \gamma<\alpha\right\}$ discussed above may help to quantitatively describe $\beta(\Gamma, \alpha)$ in terms of the trace of $\Gamma$ on the unit sphere. Apart from such a program there is also an interesting problem to understand to what extent our spherical fractional Laplacian is related to the spherical operator introduced in Ch. 8 of [Ru]. The operators turn out to be equal when $d=2$ and $\alpha=1$ (comp. Example 5.1 above with [Ru], (29.2), (29.3), page 361), but this may be more the exception than the rule.

\section{Proofs of Theorem 3.2 and 3.4}

Below we construct and prove the uniqueness of the Martin kernel $M$ at infinity for generalized cone $\Gamma$. The intersection of $\Gamma$ with the unit sphere may be highly irregular however the scaling property of the cone: $k \Gamma=\Gamma(k>0)$ allows for application of arguments similar to those used in Lemma 16 in [B1] and in [B2] for Lipschitz domains. Note that the uniqueness of the Martin kernel with the pole at a boundary point of a general domain is an open problem for our stable process. For more on this, we also refer the reader to [SW], where results on the so called "fat sets" are given.

Proof of Theorem 3.2. For $s>0$ we write $T_{s}=\tau_{\Gamma \cap\{|x|<s\}}$ and we define

$$
h_{s}(x)=\mathbf{P}_{x}\left\{X_{T_{s}} \in \Gamma\right\}, \quad x \in \mathbb{R}^{d} .
$$

By scaling we have that for all $s, t>0$ and $x \in \mathbb{R}^{d}$,

$$
h_{s}(s x)=h_{t}(t x) \quad \text { or } \quad h_{s}(x)=h_{t}\left(\frac{t}{s} x\right) .
$$


We claim there is

$$
\gamma=\gamma(\Gamma, \alpha)<\alpha
$$

and $c_{1}=c_{1}(\Gamma, \alpha)$ such that

$$
h_{1}(r \mathbf{1}) \geq c_{1} r^{\gamma}, \quad 0<r \leq 1 .
$$

The argument verifying (47) is given in the proof of Lemma 5 in [B1] and we refer the reader to that paper for details. The fact that $\gamma$ is strictly less that $\alpha$ is important and distinguishes the present situation from that of the potential theory of Brownian motion. By scaling we have $h_{s}(\mathbf{1}) \geq c_{1} s^{-\gamma}$, $s \geq 1$. We define

$$
u_{s}(x)=\frac{h_{s}(x)}{h_{s}(\mathbf{1})}, \quad s>0, x \in \mathbb{R}^{d},
$$

so that $u_{s}(\mathbf{1})=1, s>0$. Note that

$$
u_{s}(x) \leq \frac{1}{c_{1} s^{-\gamma}}=c_{1}^{-1} s^{\gamma}, \quad s \geq 1, x \in \mathbb{R}^{d} .
$$

We claim that

$$
u_{t}(x) \leq 2^{\gamma} C_{1} c_{1}^{-1}[|x| \vee 1]^{\gamma}, \quad t \geq 2, x \in \mathbb{R}^{d} .
$$

To verify (49), assume that $t \geq 2$. If $|x| \geq t / 2$ then we put $s=2(1 \vee|x|)$ and by Lemma 3.1 we have

$$
u_{t}(x) \leq C_{1} u_{s}(x) \leq C_{1} c_{1}^{-1} 2^{\gamma}[|x| \vee 1]^{\gamma} .
$$

By Harnack inequality and our normalization $u_{t}(\mathbf{1})=1$, the functions $u_{t}$ are uniformly bounded on $F$ for any compact $F \subset \Gamma$ and equicontinuous on $F$ for all large $t$. The last assertion follows from the Poisson formula for the ball or the gradient estimates of [BKN]. Therefore there is a sequence $t_{n} \rightarrow \infty$ and a function $M$ such that

$$
M(x)=\lim _{n \rightarrow \infty} u_{t_{n}}(x), \quad x \in \mathbb{R}^{d}
$$

and we take $M$ such that $M=0$ on $\Gamma^{c}$. Notice that, by (49),

$$
M(x) \leq 2^{\gamma} C_{1} c_{1}^{-1}[|x| \vee 1]^{\gamma}, \quad x \in \mathbb{R}^{d} .
$$

Let $x \in \Gamma$ and set $B=B(0, r)$, where $r>|x|$. We have

$$
u_{t_{n}}(x)=\int_{(\Gamma \cap B)^{c}} u_{t_{n}}(y) \omega_{\Gamma \cap B}^{x}(d y),
$$

where we denote by $\omega_{\Gamma \cap B}^{x}$ the $\alpha$-harmonic measure of $\Gamma \cap B$. Since $\omega_{\Gamma \cap B}^{x} \leq \omega_{B}^{x}$ on $B^{c}$ we have by (4)

$$
\omega_{\Gamma \cap B}^{x}(d y) \leq 2^{d+\alpha} 3^{-\alpha / 2} C_{\alpha}^{d} r^{\alpha}|y|^{-d-\alpha} d y, \quad|y|>2 r .
$$


Since $u_{t_{n}}(y) \rightarrow u(y)$ for all $y$, by (49), (51) and dominated convergence we

$$
M(x)=\lim _{n \rightarrow \infty} \int_{(\Gamma \cap B)^{c}} u_{t_{n}}(y) \omega_{\Gamma \cap B}^{x}(d y)=\int_{(\Gamma \cap B)^{c}} M(y) \omega_{\Gamma \cap B}^{x}(d y),
$$

proving that $M$ is a regular $\alpha$-harmonic function on $\Gamma \cap B$. We note that we used here the integrability of $|y|^{-d-\alpha+\gamma}$ at infinity, which is a consequence of (46). By the strong Markov property, $M$ is regular $\alpha$-harmonic on every open bounded subset of $\Gamma$. This proves the existence part of the Theorem.

To prove the uniqueness of $M$, we assume that there is another function $m \geq 0$ on $\mathbb{R}^{d}$ which vanishes on $\Gamma^{c}$, satisfies $m(\mathbf{1})=1$ and for which

$$
m(x)=\mathbf{E}_{x} m\left(X_{\tau_{U}}\right), \quad x \in \mathbb{R}^{d},
$$

for every open bounded $U \subset \Gamma$. By Lemma 3.1 and scaling

$$
C_{1}^{-1} m(x) \leq M(x) \leq C_{1} m(x), \quad x \in \mathbb{R}^{d} .
$$

Let $a=\inf _{x \in \Gamma} m(x) / M(x)$. For clarity, we observe that $C_{1}^{-1} \leq a \leq 1$. Let $H(x)=m(x)-a M(x)$, so that $H \geq 0$ on $\mathbb{R}^{d}$.

Assume that $H(x)>0$ for some, and therefore for every, $x \in \Gamma$. Once again by Lemma 3.1 and scaling

$$
H(x) \geq \varepsilon M(x), \quad x \in \mathbb{R}^{d},
$$

for some $\varepsilon>0$. This gives

$$
a=\inf _{x \in \Gamma} \frac{m(x)}{M(x)}=\inf _{x \in \Gamma} \frac{a M(x)+H(x)}{M(x)} \geq a+\varepsilon,
$$

which is a contradiction.

Thus $H \equiv 0$ and hence $m=a M$. The normalizing condition $m(\mathbf{1})=$ $M(\mathbf{1})=1$ yields $a=1$ and the uniqueness of $M$ is verified.

It remains to prove the homogeneity property of $M$. By the scaling of $X_{t}$, for every $k>0$ the function $M(k x) / M(k \mathbf{1})$ satisfies the hypotheses used to construct $M$. By uniqueness this function is equal to $M$, that is, $M(k x)=$ $M(x) M(k \mathbf{1})$ for $x \in \mathbb{R}^{d}$. In particular, $M(k l \mathbf{1})=M(l \mathbf{1}) M(k \mathbf{1})$ for every positive $k, l$. By continuity there exists $\beta$ such that $M(k \mathbf{1})=k^{\beta} M(\mathbf{1})=k^{\beta}$ and

$$
M(k x)=k^{\beta} M(x), \quad x \in \mathbb{R}^{d} .
$$

By (50), $M$ is locally bounded, thus $\beta \geq 0$ and $\beta \leq \gamma<\alpha$.

We claim that $\Gamma^{c}$ is non-polar if and only if 0 is a regular point of $\Gamma^{c}$, that is, $\mathbf{P}_{0}\left\{\tau_{\Gamma}^{\prime}=0\right\}=1$, where $\tau_{\Gamma}^{\prime}=\inf \left\{t>0 ; X_{t} \in \Gamma^{c}\right\}$ is the first hitting time of $\Gamma^{c}$. Indeed, it is enough to verify that if $\Gamma^{c}$ is non-polar, then $\mathbf{P}_{0}\left\{\tau_{\Gamma}^{\prime}=0\right\}=1$. But in this case $\mathbf{P}_{0}\left\{\tau_{\Gamma}^{\prime}<1 / \varepsilon\right\} \geq \varepsilon$ for some $\varepsilon>0$. By scaling of $X_{t}$ and $\Gamma$, $\mathbf{P}_{0}\left\{\tau_{\Gamma}^{\prime}=0\right\} \geq \varepsilon$. By the 0-1 law, $\mathbf{P}_{0}\left\{\tau_{\Gamma}^{\prime}=0\right\}=1$.

Consider $u(x)=\mathbf{P}_{x}\left\{X_{\tau_{\Gamma_{1}}} \in \Gamma\right\}, x \in \mathbb{R}^{d}$. If 0 is regular for $\Gamma^{c}$, then $u(x) \rightarrow 0$ as $x \rightarrow 0$. In consequence, by Lemma 3.1, $M(k \mathbf{1}) \rightarrow 0$ as $k \rightarrow 0^{+}$. 
Therefore $\beta>0$. If $\Gamma^{c}$ is polar, the indicator function of $\Gamma$ satisfies the hypotheses defining $M$ and so $\beta=0$ in this case. This completes the proof of Theorem 3.2.

We now consider $K$, the Martin kernel with the pole at 0 for the generalized cone $\Gamma \subset \mathbb{R}^{d}$. Before we prove Theorem 3.4 we note that the function $K$ as defined by (16) is bounded in the complement of every neighborhood of 0 . For dimension $d=1$ this follows by inspection of Example 3.4. For $d \geq 2$ we even have that $K(x) \rightarrow 0$ as $|x| \rightarrow \infty$.

Proof of Theorem 3.4. Consider $K$ defined by (16). Let $\eta>\varepsilon>0$ and $U=\Gamma \cap\{\eta>|y|>\varepsilon\}$. Consider an increasing sequence $\left\{U_{n}\right\}$ of open sets such that each closure $\overline{U_{n}}$ is a compact subset of $U$ and $U=\bigcup U_{n}$. Let $x \in \mathbb{R}^{d}$. Since $K$ is $\alpha$-harmonic in $\Gamma$ we have $K(x)=\mathbf{E}_{x} K\left(X_{\tau_{U_{n}}}\right), n=1,2, \ldots$ Let

$$
\begin{aligned}
& \mathcal{O}=\left\{\tau_{U_{n}}=\tau_{U} \text { for some } n\right\}, \\
& \mathcal{P}=\left\{\tau_{U_{n}}<\tau_{U} \text { for every } n\right\} .
\end{aligned}
$$

For every $n$,

$$
\begin{aligned}
K(x) & =\mathbf{E}_{x}\left\{K\left(X_{\tau_{U_{n}}}\right) ; \mathcal{O}\right\}+\mathbf{E}_{x}\left\{K\left(X_{\tau_{U_{n}}}\right) ; \mathcal{P}\right\} \\
& =\mathbf{E}_{x}\left\{K\left(X_{\tau_{U}}\right) ; \mathcal{O}, \tau_{U_{n}}=\tau_{U}\right\}+\mathbf{E}_{x}\left\{K\left(X_{\tau_{U_{n}}}\right) ; \mathcal{O}, \tau_{U_{n}}<\tau_{U}\right\} \\
& +\mathbf{E}_{x}\left\{K\left(X_{\tau_{U_{n}}}\right) ; \mathcal{P}\right\} .
\end{aligned}
$$

We have that $K$ is bounded on $U$ and continuous on $\mathbb{R}^{d}$, except for a polar subset. By monotone convergence, dominated convergence and the left continuity of the paths of $X_{t}$,

$$
K(x)=\mathbf{E}_{x}\left\{K\left(X_{\tau_{U}}\right) ; \mathcal{O}\right\}+\mathbf{E}_{x}\left\{K\left(X_{\tau_{U}}\right) ; \mathcal{P}\right\}=\mathbf{E}_{x} K\left(X_{\tau_{U}}\right) .
$$

We now let $U=\Gamma \cap\{|y|>\varepsilon\}, 0<\varepsilon<1$ and $U_{n}=\Gamma \cap\{n>|y|>\varepsilon\}$, $n=1,2 \ldots$ For these new sets $U_{n}$ we define $\mathcal{O}$ and $\mathcal{P}$ by (52) and (53), and we obtain (54). If $\alpha<d$ or $\Gamma^{c}$ is non-polar, then $K(x) \rightarrow 0$ as $|x| \rightarrow \infty$. Hence, the second and the third terms in (54) tend to 0 as $n \rightarrow \infty$. We thus obtain

$$
K(x)=\mathbf{E}_{x}\left\{K\left(X_{\tau_{U}}\right) ; \mathcal{O}\right\}=\mathbf{E}_{x}\left\{K\left(X_{\tau_{U}}\right) ; \tau_{U}<\infty\right\} .
$$

If $d=1 \leq \alpha$ and $\Gamma=\mathbb{R} \backslash\{0\}$, then $K$ is given by Example 3.4. since the process $X_{t}$ is recurrent in this case, we obtain

$$
K(x)=1=\mathbf{E}_{x} K\left(X_{\tau_{U}}\right), \text { if } x \neq 0 .
$$

We used here the observation that $X_{\tau_{U}} \neq 0 \mathbf{P}_{x}$-a.s., for $|x|>\varepsilon$. In fact, the $\mathbf{P}_{x}$ distribution of $X_{\tau_{U}}$ is absolutely continuous with respect to the Lebesgue measure on the interior of $U^{c}$, [B1].

The case of general $U$ in (17) now follows by strong Markov property. 
We now sketch a proof of uniqueness of $K$. Assume that $\tilde{K}$ is a nonnegative function on $\mathbb{R}^{d}$ such that $\tilde{K}(\mathbf{1})=1, \tilde{K}=0$ on $\Gamma^{c}$ and for every open set $U \subset \Gamma$ such that $\operatorname{dist}(0, U)>0$

$$
\tilde{K}(x)=\mathbf{E}_{x}\left\{\tilde{K}\left(X_{\tau_{U}}\right) ; \quad \tau_{U}<\infty\right\}, \quad x \in \mathbb{R}^{d} .
$$

By $[\mathrm{SW}], \tilde{K}$ is locally bounded in $\mathbb{R}^{d} \backslash\{0\}$. By (5) it is integrable in any bounded neighborhood of 0 . Given a cone $\Gamma \subset \mathbb{R}^{d}, r>0, U=\mathbb{R}^{d} \cap\{|y|>r\}$ and $A \subset \mathbb{R}^{d} \cap\{|y| \leq r\}$, we have

$$
\mathbf{P}_{x}\left\{\tau_{U \cap \Gamma}<\infty, X_{\tau_{U \cap \Gamma}} \in A\right\} \leq \mathbf{P}_{x}\left\{\tau_{U}<\infty, X_{\tau_{U}} \in A\right\}=\int_{A} \tilde{P}_{r}(x, y) d y,
$$

where $\tilde{P}_{r}(x, y)$ is given by (2). If $\alpha<d$ then

$$
\int_{\{|y|<r\}} \tilde{P}_{r}(x, y) d y \leq C|x|^{\alpha-d}, \quad x \in \mathbb{R}^{d},
$$

as can be shown from $[\mathrm{BC}]$. From this it follows that $\tilde{K}(x) \leq C|x|^{\alpha-d}$ for all sufficiently large $x$. Hence,

$$
T \tilde{K}(x)=|x|^{\alpha-d} \tilde{K}\left(x /|x|^{2}\right) \leq C .
$$

Since $\{0\}$ is polar if $\alpha<d$, it follows that $T \tilde{K}$ is regular $\alpha$-harmonic in every bounded subset of $\Gamma$. Thus $T \tilde{K}=M$ by Theorem 3.2 and so $\tilde{K}=K$.

If $d=1 \leq \alpha$ and $\Gamma=\mathbb{R}_{+}^{1}$, then a similar argument works (see [B2] for the Poisson kernel for the half-line).

If $d=1 \leq \alpha$ and $\Gamma=\mathbb{R} \backslash\{0\}$, then by (55), (2) and Harnack inequality, $\tilde{K}$ is bounded in a neighborhood of 0 , hence in $\mathbb{R}$. For $\alpha=1$ it follows that $\tilde{K}$ is regular $\alpha$-harmonic on $\Gamma$ because $\{0\}$ is polar for the Cauchy process. For $\alpha>1$, we use the Kelvin transform, and $T \tilde{K}(x) \leq c|x|^{\alpha-1}$ and this goes to 0 as $x$ goes to 0 . Thus $T \tilde{K}$ is regular $\alpha$-harmonic in every open bounded subset of $\Gamma$. By Theorem $3.2 T \tilde{K}=M$ or $\tilde{K}=K$, as before.

\section{REFERENCES}

[BLM] R. Bañuelos, R. Latała, P. J. Méndez-Hernandez, A Brascamp-Lieb-Luttinger-type inequality and applications to symmetric stable processes, Proc. Amer. Math. Soc. 129(10) (2001), 2997-3008 (electronic).

[BS] R. Bañuelos and R. Smits, Brownian motion in cones, Prob. Th. Rel. Fields, 108 (1997), 299-319.

[BDS] R. Bañuelos, R. D. DeBlassie, R. Smits, The first exit time of planar Brownian motion from the interior of a parabola, Ann. Prob. 29 (2001), 882-901.

[BC] R.F. Bass and M. Cranston, Exit times for symmetric stable processes in $\mathbb{R}^{n}$, Ann. Probab. 11(3) (1983), 578-588.

[Br] M. van den Berg, Subexponential behavior of the Dirichlet heat kernel, Preprint.

[BG] R.M. Blumenthal and R.K. Getoor, Markov Processes and Potential Theory, Springer-Verlag, New York, 1968. 
[BGR] R.M. Blumenthal, R.K. Getoor and D.B. Ray, On the distribution of first hits for the symmetric stable process, Trans. Amer. Math. Soc. 99 (1961), 540-554.

[B1] K. Bogdan, The boundary Harnack principle for the fractional Laplacian, Studia Math. 123(1) (1997), 43-80.

[B2] K. Bogdan, Representation of $\alpha$-harmonic functions in Lipschitz domains, Hiroshima Math. J. 29(2) (1999), 227-243.

[BB1] K. Bogdan and T. Byczkowski, Probabilistic proof of boundary Harnack principle for $\alpha$-harmonic functions, Potential Anal. 11 (1999), 135-156.

[BB2] K. Bogdan and T. Byczkowski, Potential theory for the $\alpha$-stable Schrödinger operator on bounded Lipschitz domains, Studia Math. 133(1) (1999), 53-92.

[BBC] K. Bogdan and K. Burdzy and Z.-Q. Chen, Censored stable processes, preprint, 2001.

[BKN] K. Bogdan, T. Kulczycki and A. Nowak, Gradient estimates for harmonic and qharmonic functions of symmetric stable processes, to appear in Ill. J. Math. (2002).

[Bd] K. Burdzy, Multidimensional Brownian excursions and potential theory, Pitman Research Notes in Mathematics 164, Longman Scientific \& Technical, 1987.

[Bk] D. L. Burkholder, Exit times of Brownian motion, harmonic majorization, and Hardy spaces, Advances in Math. 26(2) (1977), 182-205.

[CS1] Z.Q. Chen and R. Song, Estimates on Green functions and Poisson kernels for symmetric stable process, Math. Ann. 312(3) (1998), 465-501.

[CS2] Z.-Q. Chen and R. Song, Martin boundary and integral representation for harmonic functions of symmetric stable processes, J. Funct. Anal. 159(1) (1998), 267-294.

[CS3] Z.Q. Chen and R. Song, Intrinsic ultracontractivity, conditional lifetimes and conditional gauge for symmetric stable processes on rough domains, Illinois J. Math. 44(1) (2000), 138-160.

[ChZ] K. L. Chung and Z. Zhao, From Brownian motion to Schrödinger's equation, Springer-Verlag, New York, 1995.

[DZ] B. Davis and B. Zhang, Moments of the lifetime of conditioned Brownian motion in cones, Proc. Amer. Math. Soc. 121(3) (1994), 925-929.

[De1] R. DeBlassie, Exit times from cones in $R^{n}$ of Brownian motion, Prob. Th. Rel Fields., 74 (1987), 1-29.

[De2] R. DeBlassie, The first exit time of a two-dimensional symmetric stable process from a wedge, Ann. Prob., 18 (1990), 1034-1070.

[E] A. Erdélyi (Editor), Higher transcendental functions, Vol. I, II, McGraw-Hill, New York, 1953.

[IW] N. Ikeda and S. Watanabe, On some relations between the harmonic measure and the Lévy measure for a certain class of Markov processes, J. Math. Kyoto Univ. 2(1) (1962), 79-95.

[KS] J. Kelley and T. Srinivasan, Measure and integral, Vol. I, Springer-Verlag, New York, 1988.

[K1] T. Kulczycki, Properties of Green function of symmetric stable process, Probab. Math. Statist. 17(2) (1997), 339-364.

[K2] T. Kulczycki, Intrinsic ultracontractivity for symmetric stable processes, Bull. Polish Acad. Sci. Math. 46(3) (1998), 325-334.

[K3] T. Kulczycki, Exit time and Green function of cone for symmetric stable processes, Probab. Math. Statist. 19(2) (1999), 337-374.

[L] N. S. Landkof, Foundations of Modern Potential Theory, Springer-Verlag, New York, 1972.

[Li] W. Li, The first exit time of Brownian motion from unbounded domain, Preprint. 
[LS] M. Lifshitz and Z. Shi, The first exit time of Brownian motion from parabolic domain, Preprint.

$[\mathrm{M}] \quad$ P. J. Méndez-Hernández, Exit times from cones in $\mathbb{R}^{n}$ of symmetric stable processes (2000), preprint.

[Ru] B. Rubin, Fractional Integrals and Potentials, Pitman Monographs, Vol 82, Addison Wesley, 1996.

[R] M. Ryznar, Estimates of Green function for relativistic $\alpha$-stable process, Potential Anal. 17 (2002), 1-23.

[SW] R. Song and J.-M. Wu, Boundary Harnack principle for symmetric stable processes, J. Funct. Anal. 168(2) (1999), 403-427.

Department of Mathematics, Purdue University, West Lafayette, IN 47907-1395

Institute of Mathematics, Wroceaw University of Technology, 50-370 WrocŁaW, POLAND

E-mail address: banuelos@math.purdue.edu bogdan@im.pwr.wroc.pl 